\title{
Revisiting the Growth Mechanism of Hierarchical Semiconductor Nanostructures: The Role of Secondary Nucleation in Branch Formation
}

Lili Liu ${ }^{a}$, Maria L Sushko ${ }^{a}$, Edgar C Buck ${ }^{b}$, Xin Zhang ${ }^{a}$, Libor Kovarik $^{c}$, Zhizhang Shen $^{a}$, Jinhui Tao ${ }^{a}$, Elias Nakouzi ${ }^{a}$, Jun Liu ${ }^{b, d *}$ and James J De Yoreo ${ }^{a, d *}$

aPhysical and Computational Sciences Directorate, Pacific Northwest National Laboratory, Richland, WA, 99352, USA;

${ }^{b}$ Energy and Environmental Directorate, Pacific Northwest National Laboratory, Richland, WA, 99352, USA;

${ }^{c}$ Environmental Molecular Sciences Laboratory, Pacific Northwest National Laboratory,

Richland, WA, 99352, USA;

dDepartment of Materials Science and Engineering, University of Washington, Seattle,

Washington 98195, USA 

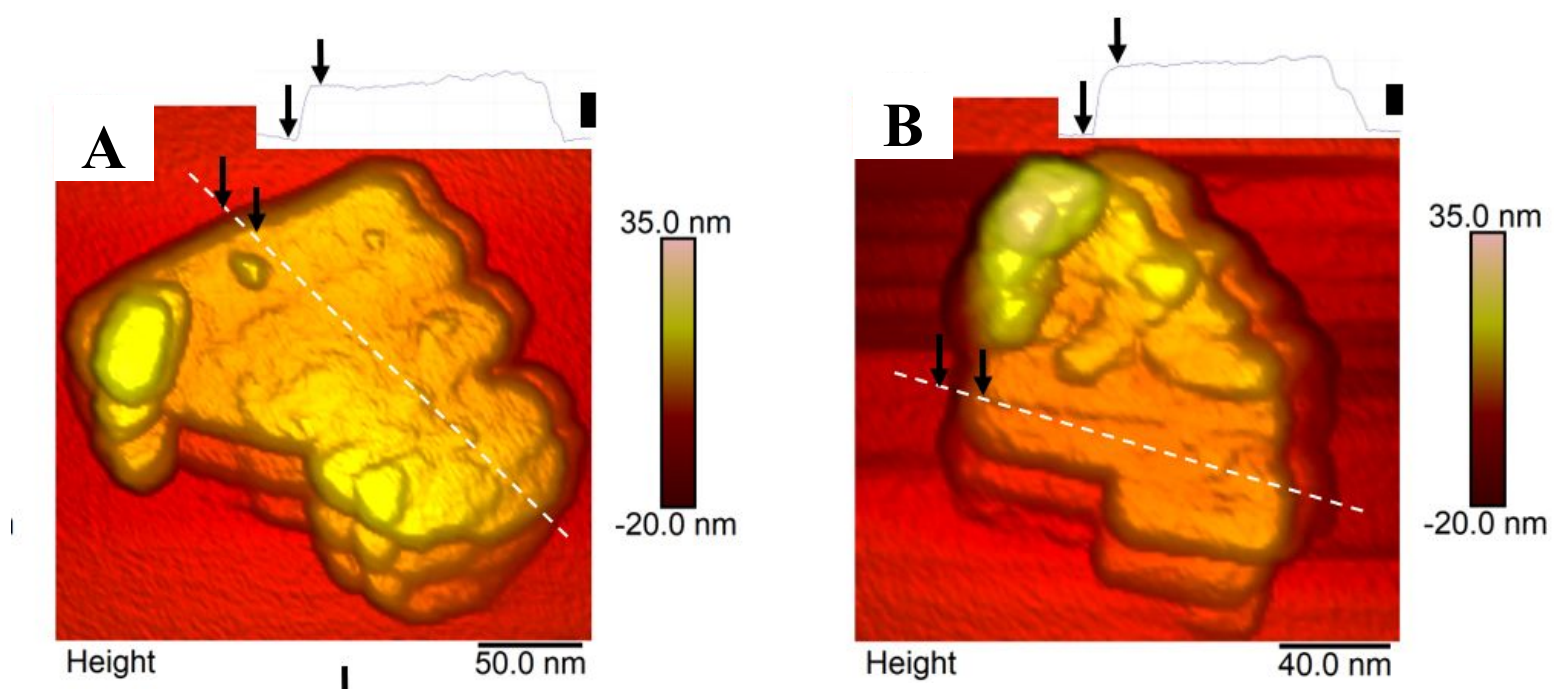

Figure S1. AFM topographical images of the typical $\mathrm{ZnO}$ platelets and cross sectional profiles noted by white dashed lines. The thicknesses are $8.6 \mathrm{~nm}$ and $12.1 \mathrm{~nm}$, respectively, measured in the locations highlighted by black arrows in (A) and (B). The vertical scale bar is $5 \mathrm{~nm}$ in (A) and (B). 

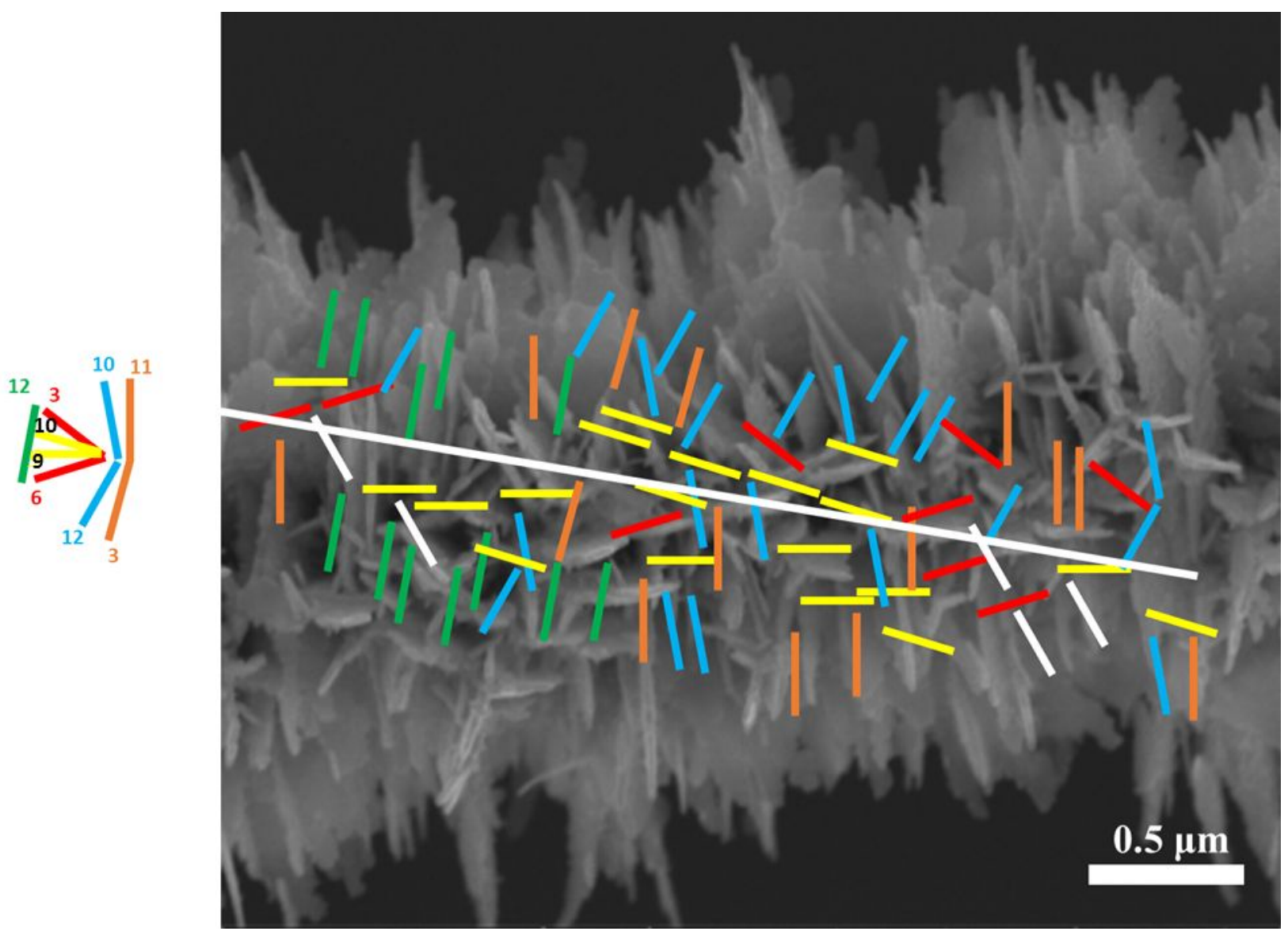

Figure S2. The non-specific crystallographic orientations relative to the stem at $5 \mathrm{mM}$ precursor concentration. The middle long white line stands for the [0001] axis of the seeds. The yellow, red, blue and orange colors represent the four distinct orientations, which are mirror reflections about the [0001] seed axis. The green yellow direction is perpendicular to the stem. The number of every individual direction is shown in the left small figure. 

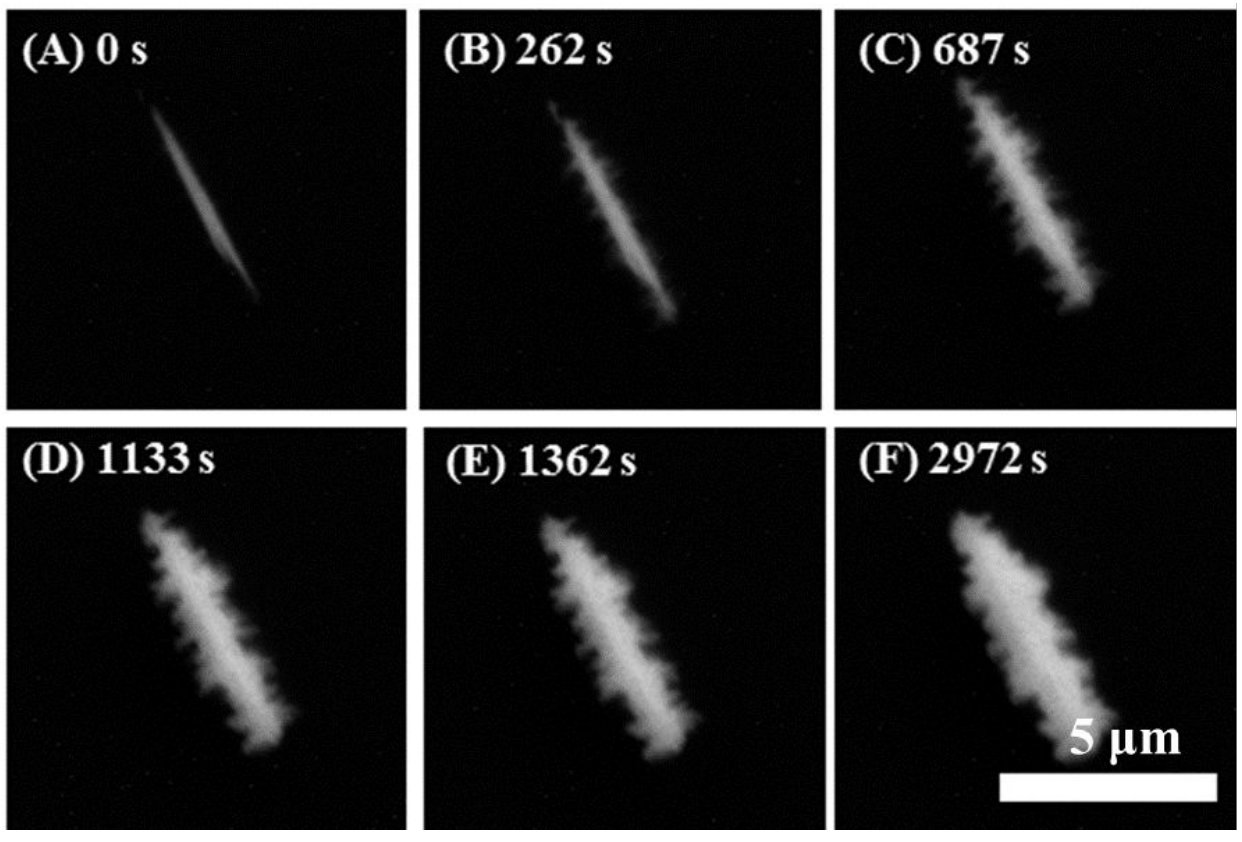

Figure S3. Time series of in situ liquid phase SEM images showing formation of branches at a mixture solution including $2 \mathrm{~mL} \mathrm{ZnO}$ rod aqueous solution, $2 \mathrm{~mL} \mathrm{Zn}\left(\mathrm{NO}_{3}\right)_{2}(5 \mathrm{mM}), 2 \mathrm{~mL} \mathrm{NaOH}$ (76.7mM), $2 \mathrm{~mL}$ PVP $(\sim 5,5000)(0.082 \mathrm{mg} / \mathrm{mL})$.
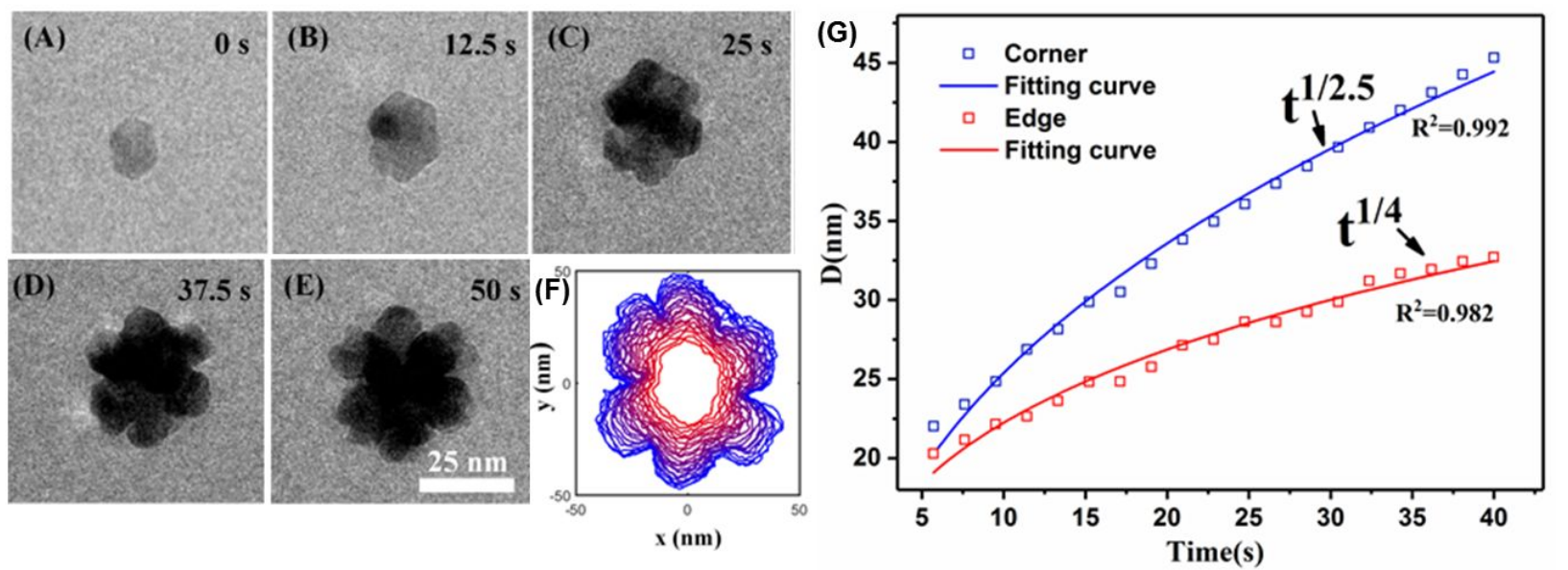

Figure S4. Time-point in-situ liquid phase TEM. (A), (B), (C), (D), (E), Scale bar is $50 \mathrm{~nm}$. (F) Contour of the $\mathrm{ZnO}$ growth drawn by the matlab software. $(\mathrm{H})$ the plotting showing particle diameter versus time. 
The time-sequential images of representative dendritic growth here is (Figure A-E) resulted from the inhomogeneous nucleation and growth under the elastic irradiation of electron beam. ${ }^{1}$ It's wellknown that the electron beam creates numerous solution radicals and molecular species, leading to the local solution reaches supersaturation. The dendritic particle trajectory was outlined in Figure $(\mathrm{F})$. Figure $(\mathrm{G})$ and $(\mathrm{H})$ show that the corner growth rate is faster than the edges growth rates(blue versus red colors). This is because the mass transparent is not consistent with the surface kinetics in the supersaturation solution, resulting the surface instability between the corner and edge that are well known to drive dendritic growth. ${ }^{2}$ So this diffusion-limited growth is particularly visualized in the precursor diffusively constrained environment of the TEM liquid cell owing to the finite size of the ultrathin liquid layer and the proximity of the $\mathrm{Si}_{3} \mathrm{~N}_{4}$ membrane. ${ }^{3}$ The result somehow provide us the diffusion-limited growth pathway rather than the particle aggregation or self-assembly.

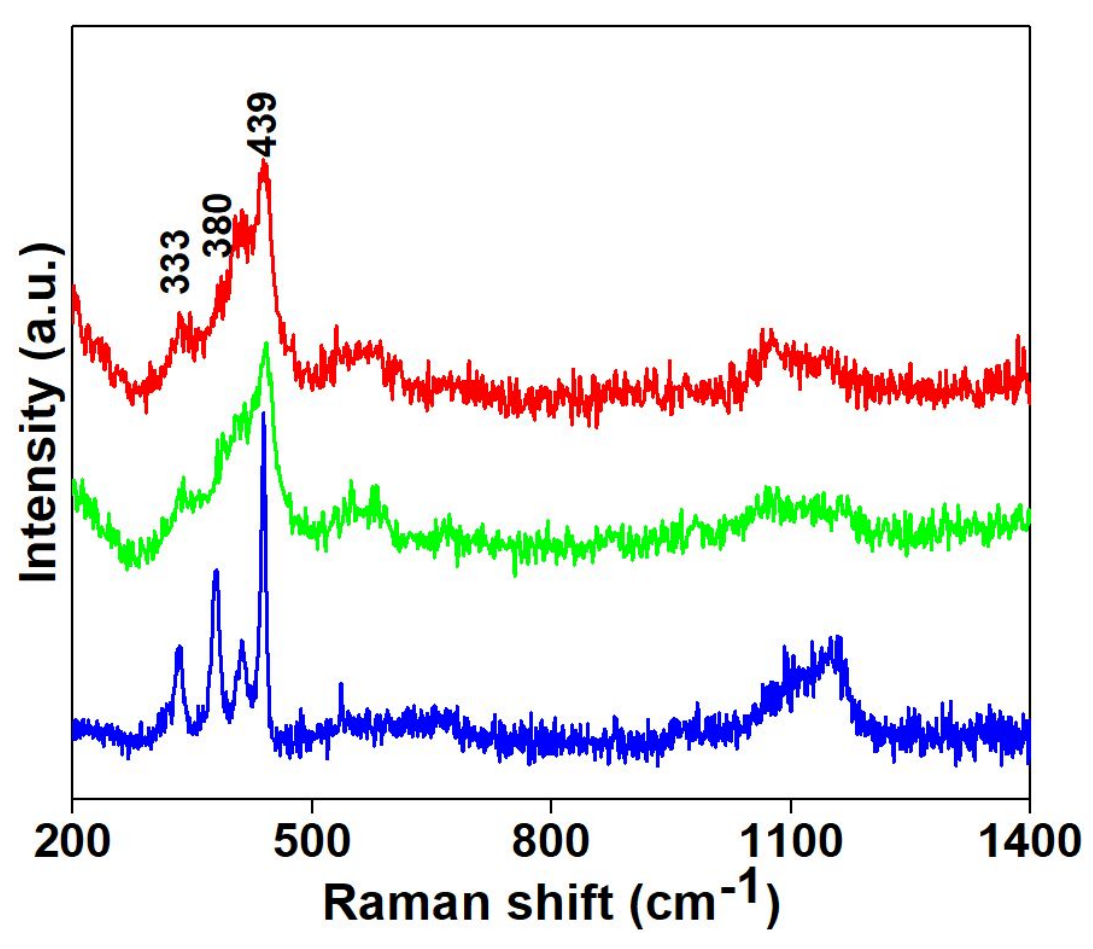

Figure S5. Typical Raman spectra of samples form in $5 \mathrm{mM} \mathrm{Zn}\left(\mathrm{NO}_{3}\right)_{2}$ precursor(red) and $10 \mathrm{mM}$ $\mathrm{Zn}\left(\mathrm{NO}_{3}\right)_{2}$ precursor (green) condition, respectively. The bottom blue curve, collected from $\mathrm{ZnO}$ single crystal with (100) face from MTI corporation, is used as a reference. 


\section{Experimental Section}

Synthesis. In every parallel experiment, we kept the ratio of $\mathrm{NaOH}$ and Polyvinylpyrrolidone (PVP) to $\mathrm{Zn}\left(\mathrm{NO}_{3}\right)_{2}$ constant. Zinc(II) stock solutions of $2 \mathrm{mM}, 5 \mathrm{mM}, 20 \mathrm{mM}, 50 \mathrm{mM}, 326 \mathrm{mM}$, and $650 \mathrm{mM}$ were freshly prepared, as well as the corresponding $\mathrm{NaOH}$ and PVP with the same ratio to $\mathrm{Zn}\left(\mathrm{NO}_{3}\right)_{2}$.

Seed-free $\mathrm{ZnO}$ synthesis: To exemplify the synthesis method, we consider $0.5 \mathrm{mM} \mathrm{Zn}\left(\mathrm{NO}_{3}\right)_{2}$ precursor. $2 \mathrm{~mL} \mathrm{Zn}\left(\mathrm{NO}_{3}\right)_{2}(5 \mathrm{mM}), 2 \mathrm{~mL} \mathrm{NaOH}(76.7 \mathrm{mM}), 2 \mathrm{~mL} \mathrm{PVP}(\sim 5,5000)(0.082 \mathrm{mg} / \mathrm{mL})$, and $12 \mathrm{~mL}$ water were mixed and aged for 24 hours at Room temperature. After completion of the reaction, the unreacted precursor and excess PVP were centrifuged and then rinsed twice with water. The obtained powder was redispersed in water for SEM and TEM characterization. Seed-mediated $\mathrm{ZnO}$ synthesis: The as-prepared $\mathrm{ZnO}$ rods obtained by $70 \mathrm{mM}$ precursor $\mathrm{Zn}$ (II) in the seed-free synthesis were re-dispersed in the aqueous solution. We consider the $0.5 \mathrm{mM}$ growth solution as an example. $2 \mathrm{~mL} \mathrm{ZnO}$ rod aqueous solution, $2 \mathrm{~mL} \mathrm{Zn}\left(\mathrm{NO}_{3}\right)_{2}(2 \mathrm{mM}), 2 \mathrm{~mL} \mathrm{NaOH}$ $(30.68 \mathrm{mM}), 2 \mathrm{~mL}$ PVP $(\sim 5,5000)(0.0328 \mathrm{mg} / \mathrm{mL})$ were mixed, and aged for 24 hours at room temperature. After completion of the reaction, the unreacted precursor and excess PVP were centrifuged and then rinsed twice with water. The obtained powder was re-dispersed in water for SEM and TEM characterization. Other concentration of growth solution was controlled similarly. All the regular SEM samples were sputter coated with a $5 \mathrm{~nm}$ layer of carbon to ensure good conductivity and imaged on a Helios NanoLab 600i SEM (FEI, Hillsboro, OR). Also, regular TEM images were conducted on FEI Titan instrument using an acceleration voltage of $300 \mathrm{kV}$.

In-situ wet cell SEM. We take the $0.5 \mathrm{mM}$ liquid cell SEM stock solution as an example. $2 \mathrm{~mL} \mathrm{ZnO}$ rod aqueous solution, $2 \mathrm{~mL} \mathrm{Zn}\left(\mathrm{NO}_{3}\right)_{2}(2 \mathrm{mM}), 2 \mathrm{~mL} \mathrm{NaOH}(30.68 \mathrm{mM}), 2 \mathrm{~mL}$ PVP $(\sim 5,5000)$ $(0.0328 \mathrm{mg} / \mathrm{mL})$ were mixed. We pipetted $3 \mu \mathrm{L}$ stock solution into the open top of a QX-202 WetSEM cell (QuantomiX), rapidly sealed the WetSEM cell and introduced it into the microscope. This operation required a duration of approximately 3 minutes.

AFM experiment. The $\mathrm{ZnO}$ platelet was cleaned in pure ethanol three times and then dispersed in ethanol by sonication for $20 \mathrm{~min}$. After that, $20 \mu \mathrm{l}$ suspension was dropped onto newly cleaved mica and dried in air spontaneously. All ex situ AFM images were captured in ScanAsyst mode at room temperature $\left(23^{\circ} \mathrm{C}\right)$ with a NanoScope 8 atomic force microscope (J scanner, Bruker) with the silicon tips (Model: Scanasyst-fluid+, triangular silicon nitride lever, $\mathrm{k}=0.7 \mathrm{~N} / \mathrm{m}$, tip radius 2 
$\mathrm{nm}$; Bruker). The force setpoint was carefully minimized to maintain the stable tracking of the topography during imaging, and the signal-to-noise ratio was maintained above 10 . The scanning speed was $0.5-1 \mathrm{~Hz}$.

In-situ liquid cell TEM. In situ liquid phase TEM (LP-TEM) experiments were carried out using a microfabricated fluid cell consisting of two silicon chips, each containing an electron transparent silicon nitride window, separated by a $100 \mathrm{~nm}$ spacer (Hummingbird Scientific, USA). 20 $\mu \mathrm{L}$ $\mathrm{Zn}\left(\mathrm{NO}_{3}\right)_{2}(5 \mathrm{mM}), 20 \mu \mathrm{L} \mathrm{NaOH}(76.7 \mathrm{mM}), 20 \mu \mathrm{L} \mathrm{PVP}(\sim 5,5000,0.082 \mathrm{mg} / \mathrm{mL})$ and $20 \mu \mathrm{LTriz}$ Base (1M) was equally mixed firstly before starting. The silicon chips were plasma-treated for $40 \mathrm{~s}$ (Harrick Plasma cleaner) to render them hydrophilic before assembling the liquid cell. The spacerchip was first placed with membrane side up inside the well-cleaned fluid stage tip, and a $0.6 \mu \mathrm{L}$ stock solution was pipetted onto its silicon nitride window. The upper chip was then placed membrane side down and aligned with the spacer-chip so that the two transparent windows could be observed in alignment under a stereomicroscope. Finally the liquid cell holder was placed in a pump station (Pfeiffer vacuum) to confirm that it was vacuum-tight before loading it into the TEM (FEI Tecnai). Bright field images and movies were recorded with an Eagle CCD detector.

Ex-situ Micro-Raman spectroscopy. ZnO (100) single crystal from MTI corporation was used as reference. The sample was synthesized in water with $5 \mathrm{mM} \mathrm{Zn}\left(\mathrm{NO}_{3}\right)_{2}$ precursor system and 10 $\mathrm{mM} \mathrm{Zn}\left(\mathrm{NO}_{3}\right)_{2}$ precursor system, respectively, followed by washing with water twice. The crystal was collected by centrifugation at $10000 \mathrm{rpm}$. The Raman spectra were collected from 50 to 1400 cm-1 under backscattering geometry by a LabRAM ARAMIS confocal Raman Microscope (HORIBA scientific, Japan) operated at a resolution of $2 \mathrm{~cm}^{-1}$ with an excitation wavelength of $532 \mathrm{~nm}$ and laser power of $2.5 \mathrm{~mW}$. A $\times 100 \mathrm{TU}$ plan fluor objective with numerical aperture of 0.90 was used to focus the sample and to collect the spectra for $10 \mathrm{~s}$.

Simulations. The focus of the theoretical simulations was on the understanding the thermodynamic driving forces for the nucleation and growth of the branches. To achieve this goal, we used a DFTbased atomistic-to-mesoscale approach we developed. ${ }^{4}$ The details of the classical density functional theory (cDFT) formulation used in this work were described in detail elsewhere ${ }^{5}$ and also provided in the Supporting Information for completeness The aqueous electrolyte solution 
was modeled as a dielectric medium with $\varepsilon=78.5$, consisting of discrete charged spherical particles representing ions, and neutral spherical particles representing water molecules. The concentration of spherical "water molecules" was $55.5 \mathrm{M}$, chosen to model experimental water density. We used experimental crystalline ionic diameters for mobile ions equal to $0.150 \mathrm{~nm}$ for $\mathrm{Zn}^{2+}$ ions, 0.204 $\mathrm{nm}$ for $\mathrm{Na}^{+}$ions and $0.358 \mathrm{~nm}$ for $\mathrm{NO}_{3}{ }^{-}$ions, and a van der Waals diameter equal to $0.275 \mathrm{~nm}$ for water molecules. The ion charges were equal to +2 for $\mathrm{Zn}^{2+}$ ions, +1 for $\mathrm{Na}^{+}$ions, -1 for $\mathrm{NO}_{3}{ }^{-}$ions, and 0 for water. Short-range interactions were treated explicitly. Experimental enthalpies of solvation were used for short-range interactions of ions with water.

Theoretical methods. The focus of the theoretical simulations was on the understanding the thermodynamic driving forces for the nucleation and growth of the branches. To achieve this goal, we used a DFT-based atomistic-to-mesoscale approach we developed. ${ }^{4-5}$ The details of the classical density functional theory (cDFT) formulation used in this work were described in detail elsewhere ${ }^{4}$ and also provided in below for completeness. The aqueous electrolyte solution was modeled as a dielectric medium with dielectric medium with $\varepsilon=78.5$, consisting of discrete charged spherical particles representing ions, and neutral spherical particles representing water molecules. The concentration of spherical "water molecules" was $55.5 \mathrm{M}$, chosen to model experimental water density. We used experimental crystalline ionic diameters for mobile ions equal to $0.150 \mathrm{~nm}$ for $\mathrm{Zn}^{2+}$ ions, $0.204 \mathrm{~nm}$ for $\mathrm{Na}^{+}$ions and $0.358 \mathrm{~nm}$ for $\mathrm{NO}_{3}^{-}$ions, and a van der Waals diameter equal to $0.275 \mathrm{~nm}$ for water molecules. The ion charges were equal to +2 for $\mathrm{Zn}^{2+}$ ions, +1 for $\mathrm{Na}^{+}$ions, -1 for $\mathrm{NO}_{3}{ }^{-}$ions, and 0 for water. Short-range interactions were treated explicitly. Experimental enthalpies of solvation were used for short-range interactions of ions with water.

cDFT simulations account only for average chemical interactions between ion and solvent species with the surface and between the species, e.g. solvation interactions, which limits the applicability of the approach to interparticle separations of no smaller than $0.5 \mathrm{~nm}$. However, as reported in our previous publications, the accuracy of our classical DFT model in calculating thermodynamic properties of electrolyte solutions, e.g. ion activity in a wide concentration range, and ion distribution at complex interfaces exceeds that of all-atom molecular dynamics with standard force-fields and the calculated results are in close agreement with state-of-the-art 
experimental data, ${ }^{6-7}$ rendering the methodology adequate for the task.

\section{Details of the classical density functional theory approach}

Classical density functional theory (cDFT) was combined with Lifshitz theory of van der Waals forces and used to evaluate forces acting between two nanoparticles immersed in electrolyte solution. The total free energy functional has the form:

$F(d)=F_{0}^{v d W}(d)+F_{h f}^{v d W}(d)+\sum F_{i}^{i o n}(d)$

where the first two terms denote zero frequency (static) and high frequency (dynamic) van der Waals interactions between two anatase nanoparticles immersed in electrolyte solution and the last term describes all ion-mediated mesoscopic and microscopic interactions described within classical density functional theory (see below) and $d$. is the distance between nanoparticle surfaces. To avoid double counting microscopic interactions, mean-field non-retarded Lifshitz theory is employed for particle/particle van der Waals terms. ${ }^{8}$ In particular, the high frequency van der Waals term is evaluated as

$F_{h f}^{v d W}(d)=\frac{k T}{8 \pi d^{2}} \sum_{n=1}^{\infty} \int_{r_{n}}^{\infty} x \log \left[\left(1-\tilde{\Delta}^{2} e^{-x}\right)\left(1-\Delta^{2} e^{-x}\right)\right] d x$

where $r_{n}=2 d \varepsilon_{w}^{1 / 2} \xi_{n} / c$ is a dimensionless factor quantifying the retardation screening and $\xi_{n}$ $=2 \pi n k T / \hbar$ defines the eigenfrequencies at which dielectric function is evaluated, $c$ is the velocity of light in vacuum and functions $\tilde{\Delta}$ and $\Delta$ are defined as

$\tilde{\Delta}=\frac{s_{w} \varepsilon_{a}\left(i \xi_{n}\right)-s_{a} \varepsilon_{w}\left(i \xi_{n}\right)}{s_{w} \varepsilon_{a}\left(i \xi_{n}\right)+s_{a} \varepsilon_{w}\left(i \xi_{n}\right)}$

$\Delta=\frac{s_{w}-s_{a}}{s_{w}+s_{a}}$

$s_{a}=\sqrt{\left(\frac{x}{r_{n}}\right)^{2}-1-\left(\frac{\varepsilon_{a}\left(i \xi_{n}\right)}{\varepsilon_{w}\left(i \xi_{n}\right)}\right)}, \quad s_{w}=\frac{x}{r_{n}}$

Note that since relative magnetic permeability of water and $\mathrm{ZnO}$ is close to $1,{ }^{9}$ we ignore the magnetic contribution in the above equation.

In the non-retarded limit $\Delta=0$ and

$\tilde{\Delta}=\frac{\varepsilon_{a}\left(i \xi_{n}\right)-\varepsilon_{w}\left(i \xi_{n}\right)}{\varepsilon_{a}\left(i \xi_{n}\right)+\varepsilon_{w}\left(i \xi_{n}\right)}$

and the high frequency van der Waals free energy is given by 
$F_{h f}^{v d W}(d)=-\frac{k T}{8 \pi d^{2}} \sum_{n=1}^{\infty} \sum_{m=1}^{\infty} \frac{\tilde{\Delta}^{2 m}\left(i \xi_{n}\right)}{m^{3}}$

The zero frequency contribution includes double-layer screening of zero frequency fluctuations and is calculated as

$$
\begin{aligned}
& F_{0}^{v d W}(d)=\frac{k T}{4 \pi} \int_{\kappa}^{\infty} \beta \log \left(1-\Delta^{2} e^{-2 \beta d}\right) d \beta \\
& \Delta \equiv \frac{\left(\sqrt{1-\left(\frac{\kappa}{\beta}\right)^{2}}\right) \varepsilon_{a}-\varepsilon_{w}}{\left(\sqrt{1-\left(\frac{\kappa}{\beta}\right)^{2}}\right) \varepsilon_{a}+\varepsilon_{w}}
\end{aligned}
$$

where $\kappa$ is the Debye screening length in electrolyte solution and $\kappa \leq \beta<\infty$.

\section{Classical Density Functional Theory}

Aqueous salt solutions are modeled as a dielectric medium with $\varepsilon_{w}=78.5$ with certain densities of positively and negatively charged, spherical particles, representing the ions and neutral spherical particles representing water molecules. The density of spherical "water molecules" was $55.5 \mathrm{M}$, which give an experimental water density. We used experimental crystalline ionic diameters for mobile ions: $\sigma_{\mathrm{Zn} 2+}=0.15 \mathrm{~nm}, \sigma_{\mathrm{NO} 3-}=0.36 \mathrm{~nm}$, and $\sigma_{\mathrm{water}}=0.275 \mathrm{~nm} .{ }^{10}$ The ion charges were $\mathrm{q}_{\mathrm{Zn} 2+}$ $=+2, \mathrm{q}_{\mathrm{NO}-}=-1$, and $\mathrm{q}_{\text {water }}=0$. All simulations were performed at $298 \mathrm{~K}$ temperature.

To determine the equilibrium water and ion distributions via cDFT and ion-mediated forces between nanoparticles, the total Helmholtz free energy functional is minimized with respect to the densities of all the species in the presence of rigid nanopraticles. For this optimization, it is convenient to partition the total free energy of the system into so-called ideal $\left(F_{i d}\right)$ and excess components $\left(\mathrm{F}_{\mathrm{ex}}\right) .{ }^{11}$ The ideal free energy corresponds to the non-interacting system and is determined by the configurational entropy contributions from water and small ions,

$F^{i d}=k T \sum_{i}^{N} \int_{\Omega}\left(\rho_{i}(\boldsymbol{r}) \log \rho_{i}(\boldsymbol{r})-\rho_{i}(\boldsymbol{r})\right) d \boldsymbol{r}$ 
where $k$ is Boltzmann's constant, $T$ is the temperature, $\rho_{i}$ is the density profile of ion and water species $i, N$ is the number of species, $\boldsymbol{r} \in \Omega$ is the ion coordinate, and $\Omega$ is the calculation domain. The excess free energy is generally not known exactly but can be approximated by $F^{e x}=F_{E D L}^{e x}+F_{h s}^{e x}+F_{e l}^{e x}+F_{h y d r}^{e x}+F_{i o n \_v d W}^{e x}$ where $F_{E D L}^{e x}=F_{C}^{e x}+F_{i m}^{e x}$ is describes first-order electrostatics and includes direct Coulomb term and image terms, $F_{h s}^{e x}$ is the hard sphere repulsion term, $F_{e l}^{e x}$ is the electrostatic ion correlation term, $F_{h y d r}^{e x}$ is the ion hydration term, and $F_{i o n \_v d W}^{e x}$ describes ion-surface van der Waals interactions.

\section{Poisson equation for first-order electrostatics}

The electric double layer contribution to the free energy $\left(F_{E D L}^{e x}\right)$ includes direct Coulomb and image interactions and is evaluated through the solution of Poisson's equation

$-\nabla \cdot \varepsilon(\boldsymbol{r}) \nabla \varphi(\boldsymbol{r})=\rho_{f}(\boldsymbol{r})+\sum_{i} q_{i} \rho_{i}(\boldsymbol{r})$ for the electrostatic potential, $\varphi(\boldsymbol{r})$, where $\square_{j}(\boldsymbol{r})$ is the fixed charge density on nanoparticle facets, $\varepsilon(\boldsymbol{r})$ is the dielectric coefficient equal to 78.5 in solution and 48 in the nanoparticles. The discrete distribution of charges on anatase facets was constructed on a 2D grid using trilinear interpolation.

\section{Fundamental Measure Theory of excluded volume effects}

Hard sphere repulsive interactions describes ion and water many-body interactions in condensed phase due to density fluctuations. These interactions were described using a Fundamental Measure theory (FMT). ${ }^{12}$ The approach is based on the solution of the Ornstein-Zernike equation for direct correlation function using the Percus-Yevick approximation and yields the following form of the corresponding component of the free energy ${ }^{13}$ :

$F_{h s}^{e x}=k T \int \Phi_{h s}\left[n_{\omega}(\boldsymbol{r})\right] d \boldsymbol{r}$

where the hard-sphere free energy density $\Phi_{h s}$ is a functional of four scalar and two vector weighted densities $\left(n_{\omega}(\boldsymbol{r})\right)$ and has the form

$$
\Phi_{h s}(r)=-n_{0} \ln \left(1-n_{3}\right)+\frac{n_{1} n_{2}}{1-n_{3}}+\left[\frac{1}{36 \pi n_{3}^{2}} \ln \left(1-n_{3}\right)+\frac{1}{36 \pi n_{3}\left(1-n_{3}\right)^{2}}\right] n_{2}^{3}
$$




$$
-\frac{\boldsymbol{n}_{1} \cdot \boldsymbol{n}_{\mathbf{2}}}{1-n_{3}}-\left[\frac{1}{12 \pi n_{3}^{2}} \ln \left(1-n_{3}\right)+\frac{1}{12 \pi n_{3}\left(1-n_{3}\right)^{2}}\right] n_{2}\left(\boldsymbol{n}_{2} \cdot \boldsymbol{n}_{2}\right)
$$

where scalar $(\alpha=0,1,2,3)$ and vector $(\beta=1,2)$ weighted densities are defined as

$n_{\alpha}(\boldsymbol{r})=\sum_{i} \int_{\Omega} \rho_{i}\left(\boldsymbol{r}^{\prime}\right) \omega_{i}^{(\alpha)}\left(\boldsymbol{r}^{\prime}-\boldsymbol{r}\right) d \boldsymbol{r}^{\prime}$

$\boldsymbol{n}_{\beta}(\boldsymbol{r})=\sum_{i} \int_{\Omega} \rho_{i}\left(\boldsymbol{r}^{\prime}\right) \boldsymbol{\omega}_{l}^{(\beta)}\left(\boldsymbol{r}^{\prime}-\boldsymbol{r}\right) d \boldsymbol{r}^{\prime}$

The "weight functions" $\omega^{(\alpha)}$ and $\omega^{(\beta)}$ characterizing the geometry of particles (hard sphere with radius $R_{i}$ for ion species $i$ ) are given by:

$\omega_{l}^{(3)}(\boldsymbol{r})=\theta\left(|\boldsymbol{r}|-R_{i}\right)$

$\omega_{i}^{(2)}(\boldsymbol{r})=\left|\nabla \theta\left(|\boldsymbol{r}|-R_{i}\right)\right|=\delta\left(|\boldsymbol{r}|-R_{i}\right)$

$\boldsymbol{\omega}_{i}^{(2)}(\boldsymbol{r})=\nabla \theta\left(|\boldsymbol{r}|-R_{i}\right)=\frac{\boldsymbol{r}}{r} \delta\left(|\boldsymbol{r}|-R_{i}\right)$

$\omega_{i}^{(0)}(\boldsymbol{r})=\omega_{i}^{(2)}(\boldsymbol{r})\left(4 \pi R_{i}^{2}\right)$

$\omega_{l}^{(1)}(\boldsymbol{r})=\omega_{l}^{(2)}(\boldsymbol{r})\left(4 \pi R_{i}\right)$

$\boldsymbol{\omega}_{i}^{(1)}(\boldsymbol{r})=\boldsymbol{\omega}_{i}^{(2)}(\boldsymbol{r})\left(4 \pi R_{i}\right)$

In the preceding formulae (70), $\theta$ is the Heaviside step function with $\theta(x)=0$ for $x>0$ and $\theta(x)=$ 1 for $x \leq 0$, and $\delta$ denotes the Dirac delta function.

\section{Mean Spherical Approximation of ion-ion electrostatic correlations}

To treat correlations resulting from electrostatic interactions between charged species on the same footing as those resulting from hard sphere excluded volume interactions Mean Spherical Approximation $^{14-15}$ was employed to solve the Ornstein-Zernike equation with respect to electrostatic direct correlation function. Taylor expansion of electrostatic free energy was cut after the second order. Then the electrostatic correlation component of the free energy $\left(F_{e l}^{e x}\right)$ is

$$
\begin{aligned}
F_{e l}^{e x}=F_{e l}^{e x}\left[\left\{\rho_{i}^{b u l k}\right\}\right]-k T \int d \boldsymbol{r} \sum_{i=+,-} \Delta C_{i}^{(1) e l}\left(\rho_{i}(\boldsymbol{r})-\rho_{i}^{b u l k}\right)- \\
\frac{k T}{2} \iint d \boldsymbol{r} d \boldsymbol{r}^{\prime} \sum_{i, j=+,-} \Delta C_{i j}^{(2) e l}\left(\left|\boldsymbol{r}-\boldsymbol{r}^{\prime}\right|\right)\left(\rho_{i}(\boldsymbol{r})-\rho_{i}^{b u l k}\right)\left(\rho_{j}\left(\boldsymbol{r}^{\prime}\right)-\rho_{j}^{b u l k}\right)
\end{aligned}
$$

where $\rho_{i}^{\text {bulk }}$ are the bulk densities of charged species and the first and second-order direct correlation functions are defined as

$\Delta C_{i}^{(1) e l}=-\mu_{i}^{e l} / k T$ 
$\Delta C_{i j}^{(2) e l}\left(\left|\boldsymbol{r}-\boldsymbol{r}^{\prime}\right|\right)=\left\{\begin{array}{lr}-\frac{q_{i} q_{j} e^{2}}{k T \varepsilon}\left[\frac{2 B}{\sigma_{i j}}-\left(\frac{2 B}{\sigma_{i j}}\right)^{2}\left(\left|\boldsymbol{r}-\boldsymbol{r}^{\prime}\right|\right)-\frac{1}{\left(\left|\boldsymbol{r}-\boldsymbol{r}^{\prime}\right|\right)}\right], & \left(\left|\boldsymbol{r}-\boldsymbol{r}^{\prime}\right|\right) \leq \sigma_{i j} \\ 0, & \left(\left|\boldsymbol{r}-\boldsymbol{r}^{\prime}\right|\right)>\sigma_{i j}\end{array}\right.$

with

$B=\left[\xi+1-(1+2 \xi)^{1 / 2}\right] / \xi$

$\xi^{2}=\kappa^{2} o_{i j}^{2}=\left[\frac{e^{2}}{\varepsilon k T} \sum_{i} q_{i}^{2} \rho_{i}^{b u l k}\right] o_{i j}^{2}$

In the above equations, $\mu_{\alpha}^{e l}$ is the chemical potential of the mobile ions, $\kappa$ is the inverse Debye length and the contact distance $\sigma_{i j}=\left(\sigma_{i}+\sigma_{j}\right) / 2$.

\section{Ion hydration interactions}

The short-range attractive hydration interactions between ions (denoted as "ion") and water "molecules" (denoted as "w") in electrolyte solution are given by

$F_{\text {sh }}^{e x}=\frac{1}{2} \int_{\Omega} \int_{\Omega} d \boldsymbol{r} d \boldsymbol{r}^{\prime} \sum_{\alpha, \beta=i o n, w} \rho_{\alpha}(\boldsymbol{r}) \rho_{\beta}\left(\boldsymbol{r}^{\prime}\right) \Phi_{\alpha \beta}\left(\left|\boldsymbol{r}-\boldsymbol{r}^{\prime}\right|\right)$ where $\Phi_{\alpha \beta}\left(\left|\boldsymbol{r}-\boldsymbol{r}^{\prime}\right|\right)$ is the square-well potential

$\Phi_{\alpha \beta}\left(\left|\boldsymbol{r}-\boldsymbol{r}^{\prime}\right|\right)=\left\{\begin{array}{c}\infty,\left|\boldsymbol{r}-\boldsymbol{r}^{\prime}\right|<\sigma_{\alpha \beta} \\ -\tau, \sigma_{\alpha \beta} \leq\left|\boldsymbol{r}-\boldsymbol{r}^{\prime}\right|<1.2 \sigma_{\alpha \beta} \\ 0,\left|\boldsymbol{r}-\boldsymbol{r}^{\prime}\right| \geq 1.2 \sigma_{\alpha \beta}\end{array}\right.$

with $\sigma_{\alpha \beta}$ equal to the contact distance between species $\square$ and $\square$ and depth $\tau$ equal to the scaled hydration enthalpy of the ions: $\tau_{C l}=0.0033166 \mathrm{eV}, \tau_{\mathrm{H}_{30} \mathrm{O}^{+}}=0.01036 \mathrm{eV}$.

\section{Ion-surface van der Waals interactions}

Lifshitz theory was used to calculate ion-surface van der Waals interactions ${ }^{16-17}$. It links ion dynamic polarizability and dielectric functions of the surface and the solvent giving the following potential for these interactions

$V_{i}^{i o n \_v d W}(d)=-\frac{\hbar}{(4 \pi)^{2} d^{3}} \int_{\Omega}^{\infty} d \xi \frac{\alpha_{i}^{*}(i \xi)}{\varepsilon_{w}(i \xi)} \varepsilon_{a}(i \xi)$

where $\alpha_{i}^{*}(i \xi)$ is the excess polarizability of ion in aqueous solution determined using time dependent DFT. 


\section{Density profiles}

Density profiles are calculated within cDFT via the minimization of the excess free energy functional $F^{e x}$ with respect to the densities of all the species. The densities satisfy the following equation

$\rho_{i}(\boldsymbol{r})=\rho_{i}^{b u l k}(\boldsymbol{r}) \exp \left(-\frac{q_{i} \varphi(\boldsymbol{r})}{k T}-\frac{1 \delta\left(F_{e l}^{e x}+F_{h s}^{e x}+F_{h y d r}^{e x}+F_{i o n_{-} v d W}^{e x}\right)}{k T}\right)$

where $\rho_{i}^{\text {bulk }}$ is the bulk value for the atomic density of matrix and minor elements in alloy and oxygen atomic density in oxide, respectively. We solve Poisson's equation for the electrostatic potential $(\varphi(\boldsymbol{r}))$. The resulting system of equations was solved iteratively to self-consistency using the numerical procedure described by Meng 5 . In particular, equilibrium ion density distributions were obtained using a relaxed Gummel iterative procedure. Convergence was considered to be achieved when the maximum difference between the input and the output density profiles between iterations was smaller than $10^{-6}$.

\section{Reference}

(1) Woehl, T. J.; Evans, J. E.; Arslan, I.; Ristenpart, W. D.; Browning, N. D., Direct in Situ Determination of the Mechanisms Controlling Nanoparticle Nucleation and Growth. ACS Nano 2012, 6, 8599-8610.

(2) Zhu, G.; Reiner, H.; Cölfen, H.; De Yoreo, J. J., Addressing some of the technical challenges associated with liquid phase S/TEM studies of particle nucleation, growth and assembly. Micron 2019, 118, 35-42.

(3) Wang, Y.; Wang, S.; Lu, X., In Situ Observation of the Growth of ZnO Nanostructures Using Liquid Cell Electron Microscopy. J. Phys. Chem. C 2017, 122, 875-879.

(4) Sushko, M. L.; Rosso, K. M., The origin of facet selectivity and alignment in anatase TiO2 nanoparticles in electrolyte solutions: implications for oriented attachment in metal oxides. Nanoscale 2016, 8, 19714-19725.

(5)Meng, D.; Zheng, B.; Lin, G.; Sushko, M. L., Numerical Solution of 3D Poisson-Nernst-Planck Equations Coupled with Classical Density Functional Theory for Modeling Ion and Electron Transport in a Confined Environment. Commun Comput Phys. 2014, 16, 1298-1322.

(6) Sushko, M. L.; Thomas, D. G.; Pabit, S. A.; Pollack, L.; Onufriev, A. V.; Baker, N. A., The Role of Correlation and Solvation in Ion Interactions with B-DNA. Biophys J 2016, 110 (2), 315 326.

(7) Sushko, M. L.; Rosso, K. M., Role of hydration forces in the properties of electrolyte solutions in the bulk and at interfaces. MRS Online Proceedings Library 2015, 1753, doi:10.1557/opl.2015.108. 
(8) Parsegian, V. A., Van der Waals Forces. Cambridge University Press: New York, NY, 2006.

(9) CRC handbook of chemistry and physics: . 2014.

(10)Marcus, Y., Ionic-Radii in Aqueous-Solutions. Chem. Rev. 1988, 88, 1475-1498.

(11)Wu, J. Z.; Li, Z. D., Density-functional theory for complex fluids. Annu. Rev. Phys. Chem. 2007, 58, 85-112.

(12)Roth, R., Fundamental measure theory for hard-sphere mixtures: a review. Journal of PhysicsCondensed Matter 2010, 22, 063102.

(13)Yu, Y. X.; Wu, J. Z., Structures of hard-sphere fluids from a modified fundamental-measure theory. J. Chem. Phys. 2002, 117, 10156-10164.

(14)Blum, L., Mean Spherical Model for Asymmetric Electrolytes .1. Method of Solution. Mol. Phys. 1975, 30, 1529-1535.

(15)Hoye, J. S.; Blum, L., Mean Spherical Model for Asymmetric Electrolytes - Thermodynamics and Pair Correlation-Function. Mol. Phys. 1978, 35, 299-300.

(16)Wernersson, E.; Kjellander, R., On the effect of image charges and ion-wall dispersion forces on electric double layer interactions. J. Chem. Phys. 2006, 125.

(17)Ninham, B. W.; Yaminsky, V., Ion binding and ion specificity: The Hofmeister effect and Onsager and Lifshitz theories. Langmuir 1997, 13, 2097-2108. 